\title{
Teneurs en phénols totaux, activités antioxydantes des macérés et décocté des feuilles de Uvaria chamae P. Beauv. (Annonaceae)
}

\author{
Bourahima BAMBA ${ }^{1 *}$, Comoé Koffi Donatien BENIE ${ }^{1}$, Abou OUATTARA ${ }^{2}$, Dahiro Noël \\ DOUKOUROU $^{1}$, Richard Kamou KAMOU ${ }^{1}$ et Karamoko OUATTARA ${ }^{1}$ \\ ${ }^{1}$ Laboratoire de Pharmacodynamie Biochimique, UFR Biosciences, Université Félix Houphouët-Boigny, \\ Abidjan Côte d'Ivoire, 22 BP 582 Abidjan 22. \\ ${ }^{2}$ Unité de Formation et de Recherche en Agroforesterie, Département de Biochimie-Microbiologie, Université \\ Jean Lorougnon Guédé, BP 150 Daloa, Côte d'Ivoire. \\ *Auteur correspondant ; E-mail: bourahimabamba@yahoo.fr; Tel: +225 0707164077, +2250506598905
}

\author{
Received: 28-09-2020 $\quad$ Accepted: 19-02-2021 $\quad$ Published: 28-02-2021
}

\section{RESUME}

Uvaria chamae est une plante de la biodiversité Ivoirienne utilisée en médecine traditionnelle. Malgré les vertus biologiques et thérapeutiques de cette plante, peu d'informations sur la teneur en phénols totaux et l'activité antioxydante existent. L'objectif de cette étude était d'évaluer la teneur en phénols totaux, l'activité antioxydante de macéré aqueux, de macéré éthanolique et de décocté aqueux des feuilles de Uvaria chamae. La teneur en phénols totaux a été effectuée par la méthode de Folin-Ciocalteu. L'activité antioxydante a été évaluée par la méthode de piégeage du DPPH (2,2-diphenyl-1-picrylhydrazyle) et d'ORAC (Oxygen Radical Absorbance Capacity). La teneur en phénols totaux était respectivement de 110,51 \pm 7,18 $\mu \mathrm{g}$ GAE/mg d'extraits, de 86,34 \pm $1,38 \mu \mathrm{g} \mathrm{GAE} / \mathrm{mg}$ d'extrait et de 65,80 $\pm 0,13 \mu \mathrm{g}$ GAE$/ \mathrm{mg}$ d'extrait pour le macéré éthanolique, le décocté aqueux et le macéré aqueux de Uvaria chamae. Les différents macérés et décocté ont présenté une activité de piégeage des deux radicaux libres. S'agissant du radical DPPH, le macéré éthanolique de $U$. chamae exerce une forte activité antiradicalaire significative avec un CI50 de l'ordre de $0,08 \pm 0,02 \mathrm{mg} / \mathrm{ml}$. Avec la méthode ORAC, le macéré éthanolique de $U$. chamae $(3,51 \pm 0,15 \mu \mathrm{mol} \mathrm{TE} / \mu \mathrm{g}$ d'extrait) et le décocté aqueux de $U$. chamae $(3,36$ $\pm 0,18 \mu \mathrm{mol} \mathrm{TE} / \mu \mathrm{g}$ d'extrait) présentent des valeurs d'activités antioxydantes significatives. Par contre, les valeurs de l'activité antioxydante du macéré aqueux de $U$. chamae $(3,04 \pm 0,20 \mu \mathrm{mol} \mathrm{TE} / \mu \mathrm{g}$ d'extrait $)$ et du témoin positif : extrait éthanolique de $R$. officinalis $(2,54 \pm 0,06 \mu \mathrm{mol} \mathrm{TE} / \mu \mathrm{g}$ d'extrait) ne sont pas significativement différentes $(\mathrm{P}>0,05)$. Ces résultats montrent l'existence d'une bonne corrélation linéaire entre la teneur en phénols totaux et le pouvoir antioxydant des différents macérés et décocté de cette plante. Cette plante Ivoirienne pourrait donc être considérée comme une source d'antioxydant naturel à des fins médicinales. (C) 2021 International Formulae Group. All rights reserved.

Mots clés : Uvaria chamae, teneur en phénols totaux, antioxydant, Côte d'Ivoire.

\section{Total phenol content, antioxidant activities of macerated and decocted leaves of Uvaria chamae P. Beauv. (Annonaceae)}

\begin{abstract}
Uvaria chamae is a plant of the Ivorian biodiversity used in traditional medicine. Despite the biological and therapeutic virtues of this plant, little information on the total phenol content and antioxidant activity exist. The objective of this study was to evaluate the total phenol content, antioxidant activity of aqueous macerate,
\end{abstract}


ethanolic macerate and aqueous decocate of Uvaria chamae leaves. The total phenol content was determined by the Folin-Ciocalteu method. Antioxidant activity was evaluated by the DPPH (2,2-diphenyl-1-picrylhydrazyl) and ORAC (Oxygen Radical Absorbance Capacity) scavenging method. The total phenol content was $110.51 \pm$ $7.18 \mu \mathrm{g}$ GAE$/ \mathrm{mg}$ extract, $86.34 \pm 1.38 \mu \mathrm{g}$ GAE$/ \mathrm{mg}$ extract and $65.80 \pm 0.13 \mu \mathrm{g} \mathrm{GAE} / \mathrm{mg}$ extract for ethanolic macerate, aqueous decocate and aqueous macerate of Uvaria chamae, respectively. The different macerated and decocted products presented an activity of scavenging of the two free radicals. Concerning the DPPH radical, the ethanolic macerate of $U$. chamae exerts a significant antiradical activity with an IC50 of the order of $0.08 \pm 0.02$ $\mathrm{mg} / \mathrm{ml}$. With the ORAC method, the ethanolic macerate of $U$. chamae $(3.51 \pm 0.15 \mu \mathrm{mol} \mathrm{TE} / \mu \mathrm{g}$ extract $)$ and the aqueous decocate of $U$. chamae $(3.36 \pm 0.18 \mu \mathrm{mol} \mathrm{TE} / \mu \mathrm{g}$ extract) show significant antioxidant activity values. On the other hand, the antioxidant activity values of the aqueous macerate of $U$. chamae $(3.04 \pm 0.20 \mu \mathrm{mol} \mathrm{TE} / \mu \mathrm{g}$ extract) and the positive control: ethanolic extract of $R$. officinalis $(2.54 \pm 0.06 \mu \mathrm{mol} \mathrm{TE} / \mu \mathrm{g}$ extract $)$ are not significantly different $(\mathrm{P}>0.05)$. These results show the existence of a good linear correlation between the total phenol content and the antioxidant power of the different macerated and decocted phenols of this plant. This Ivorian plant could thus be considered as a source of natural antioxidant for medicinal purposes.

(C) 2021 International Formulae Group. All rights reserved.

Keywords : Uvaria chamae, total phenol content, antioxidant, Côte d'Ivoire.

\section{INTRODUCTION}

$\mathrm{Au}$ cours de son fonctionnement, l'organisme produit en permanence et en faible quantité des espèces chimiques radicalaires. Ces radicaux libres, bénéfiques à l'organisme (à faible doses), sont produits par divers mécanismes physiologiques.

En effet, ces mécanismes physiologiques concernent les réactions énergétiques, de défense réalisées respectivement par la mitochondrie et les macrophages ainsi que les réactions de médiation tissulaires. Cette production physiologique est maîtrisée par des systèmes antioxydants réalisant une adaptation par rapport aux taux d'oxydants présents.

Dans ces conditions, la balance antioxydants/pro-oxydants est donc en équilibre. En cas de déséquilibre soit par déficit en antioxydants ou par excès d'oxydants (endogènes ou exogènes), l'excès de ces radicaux libres est appelé stress oxydant (Favier, 2003). Ce stress oxydatif peut générer des dommages oxydatifs se traduisant par diverses altérations biochimiques intracellulaires telles que l'oxydation de l'ADN, des protéines, et des lipides (Favier, 2003 ; Davies, 2004 ; Niki, 2009 ; Sanchez, 2017). Il est impliqué dans la genèse de nombreuses pathologies telles que le diabète, l'obésité, le cancer, l'inflammation et les maladies dégénératives (Maiese et al., 2007 ;
Vincent et al., 2007; Melo et al., 2011; Sanchez, 2017).

Par ailleurs, la maîtrise de ces pathologies devient complexe. Cependant, le traitement actuel des maladies liées aux stress oxydatifs fait appel à des antioxydants synthétiques. Ces molécules bien qu'étant efficaces présentent le plus souvent des effets néfastes pour l'organisme (Panicker et al., 2014).

Il est donc important de trouver une alternative à l'utilisation de ces médicaments synthétiques antioxydants à effets néfastes. De ce fait, le contrôle du stress oxydatif constitue un enjeu majeur de santé publique nécessitant la recherche de composés d'origine végétales aux propriétés thérapeutiques accessibles à tous.

Parmi ces composés d'origine végétales, Uvaria chamae est une plante de la biodiversité Ivoirienne utilisée en médecine traditionnelle pour le traitement de nombreuses pathologies comme les hémorragies, la dysenterie, les plaies, le mal de gorge, les douleurs abdominales, la fatigue, les troubles respiratoires et l'anémie (Adomou et al., 2012 ; Koné et al., 2013). Le jus des feuilles est également utilisé comme une lotion en application locale pour traiter les œdèmes, les blessures, la conjonctivite et l'uvéite. Les racines sont recommandées pour le traitement des maladies cardiaques, la fièvre et les 
saignements du nez (Etukudo, 2003 ; Moses et al., 2013). Aussi, des propriétés antioxydantes ont-elles été rapportées par Koné et al. (2015) et l'effet anti-inflammatoire de cet extrait inhiberait significativement l'œdème de la patte de rats induit par la carragénine (Bamba et al., 2019).

L'intérêt de cette plante pour la médecine traditionnelle et la recherche de nouvelles molécules bioactives, réside dans le fait qu'elle est largement utilisée par la population Africaine pour le traitement des maladies oxydatives.

De plus, malgré quelques études réalisées sur les feuilles de $U$. chamae, les données sur la teneur en phénols totaux et l'activité antioxydante peuvant également définir l'intérêt médical potentiel de cette plante restent insuffisantes.

L'objectif de cette étude était d'évaluer la teneur en phénols totaux ainsi que l'activité antioxydante de macéré aqueux, de macéré éthanolique et de décocté aqueux des feuilles de Uvaria chamae.

\section{MATERIEL ET METHODES}

\section{Matériel végétal}

Le matériel végétal utilisé était constitué des feuilles de Uvaria chamae. Ces feuilles ont été récoltées à M'BENGUE, une sous-préfecture située au Nord de la Côte d'Ivoire dans la région du Poro dont KORHOGO est le chef-lieu. La récolte de cette plante a été effectuée au mois d'Octobre 2016 à la suite d'une enquête ethnobotanique et authentifiée par le Centre National Floristique (CNF) de l'Université Félix Houphouët Boigny (Côte d'Ivoire).

\section{Préparation de macéré aqueux, macéré éthanolique et de décocté aqueux des feuilles de Uvaria chamae \\ Broyage}

Apres lavage à l'eau distillée, les feuilles de Uvaria chamae découpées en petits morceaux ont été séchées pendant trois semaines à la température ambiante $\left(25^{\circ} \mathrm{C}\right.$ à 30 ${ }^{\circ} \mathrm{C}$ ) à l'abri du soleil pour mieux conserver les molécules sensibles à la chaleur et à la lumière.
Ces feuilles séchées ont été broyées en poudre fine à l'aide d'un mortier traditionnel. La poudre obtenue a été stockée dans un flacon hermétique. Cette poudre a servi à la préparation des différents macérés et décocté.

\section{Préparation de macéré aqueux et macéré éthanolique $96 \%$}

Le macéré aqueux et éthanolique $96 \%$ des feuilles de Uvaria chamae ont été préparé selon la méthode d'Olakunle et al. (2005) avec de légères modifications. Une masse de cent grammes $(100 \mathrm{~g})$ de poudre de cette plante a été macérée dans 1 litre d'eau distillée sous agitation au blinder $\left(\mathrm{Nasco}^{\circledR}\right.$, Chine) pour le macéré aqueux. Quant au macéré éthanolique $96 \%$, c'est une masse de vingt grammes (20 g) de poudre qui a été macérée dans $160 \mathrm{ml}$ d'éthanol (96\%).

Ensuite, le mélange de chaque macéré a été essoré dans un carré de tissu propre, filtré successivement deux fois sur du coton hydrophile et une fois sur du papier filtre (papier Whatman ${ }^{\circledR} 3 \mathrm{~mm}$ ). Ce filtrat a ensuite été séché lentement à l'étuve à $50{ }^{\circ} \mathrm{C}$ pendant 72 heures pour chaque macéré.

En fin, la poudre obtenue (macéré total aqueux ou macéré éthanolique 96\%) a été stockée dans un bocal à fermeture hermétique et conservée au réfrigérateur à $+4{ }^{\circ} \mathrm{C}$ (Zirihi et al., 2003).

\section{Préparation de décocté aqueux}

Une masse de $100 \mathrm{~g}$ de poudre végétale fine a été ajoutée à $1 \mathrm{~L}$ d'eau distillée et le mélange est porté à ébullition pendant 15 minutes. Après refroidissement, la décoction (le mélange) a été essorée dans un carré de tissu propre, puis filtrée successivement deux fois sur du coton hydrophile et une fois sur du papier filtre (papier Whatman ${ }^{\circledR} 3 \mathrm{~mm}$ ). Le filtrat obtenu a été ensuite séché lentement à l'étuve à $50{ }^{\circ} \mathrm{C}$ pendant $72 \mathrm{~h}$. La poudre obtenue ou décocté aqueux a été conservée dans un flacon stérile et sec.

\section{Dosage des phénols totaux}

Les phénols totaux et d'autres composés réducteurs dans le macéré aqueux, le macéré éthanolique et le décocté de Uvaria 
chamae ont été quantifiés selon la méthode de Folin-ciocalteu décrite par Morel et al. (2018) avec quelques modifications.

Les extraits testés et l'extrait éthanolique de Romarin officinalis (Contrôle positif) ont été solubilisés dans du DMSO (4 $\mathrm{mg} / \mathrm{mL}$ ) puis dilués à différentes concentrations $(1 ; 0,5 ; 0,25$ et $0,125 \mathrm{mg} / \mathrm{mL})$ dans de l'eau distillée. Une gamme d'acide gallique $(1,56 ; 3,125 ; 6,25 ; 12,5 ; 25 ; 50$ et $75 \mu \mathrm{g} / \mathrm{mL}$ ) a été réalisée dans de l'eau distillée.

Dans des plaques à 96 puits ont été repartis en triplicate des aliquotes de $50 \mu \mathrm{L}$ d'extraits testés, d'acide gallique et de l'extrait de Romarin officinalis pour chaque concentration testée. Des aliquotes de $50 \mu \mathrm{L}$ de l'eau distillée puis $50 \mu \mathrm{L}$ de réactif de folinciocalteu à $10 \%$ et $50 \mu \mathrm{L}$ de la solution de carbonate de sodium à $1 \mathrm{M}$ ont été respectivement ajoutés à chaque puits. Les plaques ont été incubées à la température ambiante et à l'abri de la lumière avec une lente agitation continue pendant 60 minutes.

Une courbe d'étalonnage a été établie avec de l'acide gallique. L'absorbance a été mesurée avec un lecteur de plaques de Microtitration Moléculaire Devices UV Max à $650 \mathrm{~nm}$. Les résultats sont exprimés en microgrammes d'équivalents d'acide gallique par milligramme d'extrait ( $\mu \mathrm{g}$ GAE/mg d'extrait).

\section{Activité antioxydante}

\section{Mesures de l'activité antiradicalaire (DPPH)}

Les mesures des activités

antiradicalaires du macéré aqueux, du macéré éthanolique et du décocté aqueux de Uvaria chamae ont été effectuées par le test au 2,2diphényl-1-picrylhydrazyl (DPPH) selon la méthode décrite par Morel et al. (2018) avec quelques modifications.

Les extraits testés ont été solubilisés dans du DMSO et dilués à différentes concentrations $(0,5 ; 0,25$ et $0,125 \mathrm{mg} / \mathrm{mL})$ à partir de la solution mère à $1 \mathrm{mg} / \mathrm{mL}$ dans de l'éthanol absolu. L'éthanol absolu a été utilisé comme blanc en parallèles avec les extraits testés. Une gamme de Trolox (analogue hydrophile de l' $\alpha$-tocophérol) à $100 ; 75 ; 50$; $25 ; 12,5$ et $0 \mu \mathrm{M}$ a été réalisée pour l'étalonnage. L'acide chlorogénique $(0,01$ $\mathrm{mg} / \mathrm{mL}$ ) et l'extrait éthanolique de Romarin officinalis $(0,2 \mathrm{mg} / \mathrm{mL})$ ont été utilisés comme témoins positifs.

Dans des plaques à 96 puits ont été repartis en triplicate des aliquotes de $100 \mu \mathrm{lde}$ ces solutions, pour chaque concentration testée. Ensuite, une solution de $75 \mu \mathrm{L}$ d'éthanol absolu a été ajoutée. La réaction a été initiée en ajoutant $25 \mu \mathrm{L}$ d'une solution de DPPH fraîchement préparée pour obtenir un volume final de $200 \mu 1$.

Après 30 minutes dans l'obscurité et à température ambiante, l'absorbance a été mesurée à $550 \mathrm{~nm}$. Les activités antiradicalaires des extraits végétaux testés ont été comparées à la courbe d'étalonnage de Trolox. Les résultats ont été exprimés en équivalents Trolox ( $\mu$ mol d'équivalents Trolox par gramme d'extrait). Ils ont été également exprimés en concentration d'extrait végétal responsable de $50 \%$ d'inhibition des radicaux DPPH $\left(\mathrm{CI}_{50}\right.$ en $\left.\mathrm{mg} / \mathrm{ml}\right)$.

\section{Mesures de l'ORAC (Oxygen Radical Antioxydant Capacity)}

Le test ORAC est un test pour évaluer le pouvoir des composés présents dans un extrait pouvant réduire les radicaux peroxyles. Ce test consiste à suivre la perte de la fluorescence de la fluorescéine qui est induite par un générateur de radical peroxylé : le 2-2-Azobis (2amidinopropane) dihydrochloride (AAPH).

Le dosage de ORAC a été réalisé selon la méthode décrite par Morel et al. (2018) avec quelques modifications. Une solution de tampon phosphate à $75 \mathrm{mM}(\mathrm{pH} \mathrm{7,4)}$ a été réalisée. Une gamme de Trolox à $75 ; 50 ; 25$; 12,$5 ; 6,25$ et $0 \mu \mathrm{M}$ a été réalisée dans du tampon phosphate pour l'étalonnage. L'acide chlorogénique $(8,8 \mu \mathrm{M})$ et l'extrait éthanolique de Romarin officinalis $(12,5 \mu \mathrm{g} / \mathrm{mL})$ ont été utilisés comme témoin positif

Les extraits végétaux ont été solubilisés dans du DMSO et dilués à différentes concentrations $(25 \mu \mathrm{g} / \mathrm{mL}, 12,5 \mu \mathrm{g} / \mathrm{mL}, 6,25$ $\mu \mathrm{g} / \mathrm{mL}$ et $3,125 \mu \mathrm{g} / \mathrm{mL}$ ) à partir de la solution mère de $1 \mathrm{mg} / \mathrm{mL}$ dans du tampon phosphate. Un volume de $20 \mu \mathrm{L}$ de chaque solution (gamme de Trolox, acide chlorogénique, extrait éthanolique de $R$. officinalis, extraits des plantes) ont été distribués dans une plaque de 96 puits en triplicate. Un volume de $100 \mu \mathrm{L}$ de 
tampon phosphate et $100 \mu \mathrm{L}$ de fluorescéine $(0,1 \mu \mathrm{M}$ dans un tampon phosphate) ont été rajoutés à chaque puits. La fluorescéine a été préparée juste après dépôt des solutions précédentes dans les puits. A l'abris de la lumière, la plaque a été pré incubée à $37{ }^{\circ} \mathrm{C}$ pendant $10 \mathrm{~min}$ sous une lente agitation mécanique.

La réaction a été initiée en ajoutant 50 $\mu \mathrm{L}$ de l'AAPH fraîchement préparée (103,2 $\mathrm{mg} / \mathrm{mL}$ dans un tampon phosphate). La fluorescence a été mesurée et enregistrée toutes les 5 min pendant $70 \mathrm{~min}$ à une longueur d'onde d'excitation de $485 \mathrm{~nm}$ et $520 \mathrm{~nm}$ d'émission à l'aide d'un spectrophotomètre de type Berthold (Berthold Technologies, Bad Wildbad, Tristar, LP941, Germany).

Les valeurs de l'ORAC ont été calculées à l'aide d'une équation de régression entre la concentration de Trolox et l'aire nette sous la courbe de décroissance de la fluorescéine. Elles ont été exprimées en $\mu \mathrm{mol}$ équivalents Trolox par microgramme d'extrait $\sec (\mu \mathrm{mol} \mathrm{TE} / \mu \mathrm{g}$ d'extrait sec).

\section{Analyses statistiques}

Les résultats des tests effectués sont exprimés en moyenne $\pm \mathrm{SD}$. La représentation graphique des données a été effectuée à partir du logiciel Graph Pad Prism 5.0 (Microsoft U.S.A). Ces résultats sont également analysés par le test d'ANOVA one way suivi du test Bonferroni pour les comparaisons multiples et la détermination des taux de signification. Les valeurs de $\mathrm{P} \leq 0,05$ sont considérées statistiquement significatives.

\section{RESULTATS}

\section{Dosage des phénols totaux}

Les résultats obtenus sont exprimés en microgramme équivalent d'acide gallique par milligramme d'extrait ( $\mu \mathrm{g} \mathrm{GAE} / \mathrm{mg}$ d'extrait), en utilisant l'équation linéaire $\mathrm{y}=\mathrm{ax}+\mathrm{b}$ de la courbe d'étalonnage tracée avec des solutions d'étalon de l'acide gallique à différentes concentrations (Figure 1).

La Figure 2 montre les teneurs en phénols totaux de macéré aqueux, de macéré éthanolique et de décocté aqueux de U. chamae ainsi que de l'extrait éthanolique de Rosmarinus Officinalis analysé. L'analyse de ces résultats montre que les teneurs en phénols totaux varient de $60,09 \pm 1,90 \mu \mathrm{g} \mathrm{GAE} / \mathrm{mg}$ d'extrait à $110,51 \pm 7,18 \mu \mathrm{g}$ GAE/mg d'extrait dans les extraits étudiés. Ainsi l'extrait éthanolique de $R$. officinalis (témoin positif) présente la valeur la plus faible avec 60,09 \pm $1,90 \mu \mathrm{g} \mathrm{GAE} / \mathrm{mg}$ d'extrait alors que le macéré éthanolique de $U$. chamae possède la plus grande valeur avec 110,51 $\pm 7,18 \mu \mathrm{g} \mathrm{GAE} / \mathrm{mg}$ d'extrait.

Quant aux macérés aqueux et décocté aqueux de $U$. chamae, ils enregistrent des valeurs de 65,80 $\pm 0,13 \mu \mathrm{g} \mathrm{GAE} / \mathrm{mg}$ d'extrait et de $86,34 \pm 1,38 \mu \mathrm{g}$ GAE $/ \mathrm{mg}$ d'extrait, respectivement. L'analyse statistique ne montre pas de différence significative $(\mathrm{P}>$ $0,05)$ entre le macéré aqueux de $U$. chamae et l'extrait éthanolique de $R$. officinalis. Par contre, il existe une différence significative ( $\mathrm{P}$ $<0,05)$ d'une part, entre le macéré éthanolique, le décocté aqueux de $U$. chamae et l'extrait éthanolique de $R$. officinalis, et d'autre part, entre les différents macérés et décocté de $U$. chamae (Figure 2).

\section{Activité antioxydante \\ Mesure de l'activité antiradicalaire (DPPH)}

Les résultats de l'évaluation de l'activité antioxydante des extraits étudiés par le test de DPPH sont exprimés par la concentration en équivalents Trolox par gramme d'extrait sec et en concentration inhibitrice $50 \%$ du radical DPPH par les extraits. Ces résultats sont résumés dans le Tableau 1 et sur la Figure 3.

L'ensemble des extraits étudiés révèlent des activités antiradicalaires qui se manifestent par de fortes valeurs en concentrations équivalents Trolox. Ces concentrations varient de $203,14 \pm 15,90$ à $371,26 \pm 18,42 \mu \mathrm{mol} \mathrm{TE} / \mathrm{g}$ d'extrait. Le macéré éthanolique de $U$. chamae (371,26 $\pm 18,42 \mu \mathrm{mol} \mathrm{TE} / \mathrm{g}$ d'extrait) présente un très bon pouvoir antioxydant efficace, significativement $(\mathrm{P}<0,05)$ supérieur à celui de l'extrait éthanolique de $R$. officinalis (antioxydant de référence) avec 261,30 \pm 18,12 $\mu \mathrm{mol} \mathrm{TE} / \mathrm{g}$ d'extrait.

Par contre, le macéré aqueux de $U$. chamae $(203,14 \pm 15,90 \mu \mathrm{mol}$ TE/g d'extrait) et le décocté aqueux de $U$. chamae $(224,39 \pm$ $18,39 \mu \mathrm{mol} \mathrm{TE} / \mathrm{g}$ d'extrait) ont enregistré des 
valeurs antioxydantes inférieures mais non significativement différentes de celle de l'extrait éthanolique de $R$ officinalis (261,30 \pm 18,12 $\mu \mathrm{mol}$ TE/g d'extrait) (Figure 3). Cependant, l'acide chlorogénique a indiqué la plus forte valeur antiradicalaire avec 3236,57 \pm 305,97 $\mu \mathrm{mol} \mathrm{TE} / \mathrm{g}$ d'extrait (Tableau 1).

Le Tableau 1 révèle que les valeurs des CI50 des extraits varient entre $0,08 \pm 0,02$ $\mathrm{mg} / \mathrm{ml}$ à des valeurs supérieures à $1 \mathrm{mg} / \mathrm{ml}$. Le macéré éthanolique de $U$. chamae exerce une forte activité antioxydante significative avec un CI50 de l'ordre de 0,08 $\pm 0,02 \mathrm{mg} / \mathrm{mL}$. Ce macéré éthanolique a donc présenté une activité antioxydante très significative et supérieure à celle de l'extrait éthanolique de $R$. officinalis.

Par contre, le macéré aqueux de $U$. chamae ainsi que le décocté aqueux de $U$. chamae présentent des activités antioxydantes moins significatives $(\mathrm{P}>0,05)$ avec des valeurs de CI50 supérieurs à $1 \mathrm{mg} / \mathrm{ml}$ (Tableau 1).

- Corrélation entre teneur en phénols totaux et activité antioxydante (DPPH)

Les résultats obtenus montrent une bonne corrélation linéaire $\left(\mathrm{R}^{2}\right)$ entre la teneur en phénols totaux et le pouvoir antiradicalaire des extraits des feuilles de $U$. chamae à raison de 0,8755 (Figure 4).

Mesure de ORAC (Oxygen Radical Absorbance Capacity)

Le macéré éthanolique de $U$. chamae $(3,51 \pm 0,15 \mu \mathrm{mol} \mathrm{TE} / \mu \mathrm{g}$ d'extrait) et le décocté aqueux de $U$. chamae $(3,36 \pm 0,18$ $\mu$ mol TE/ $\mu \mathrm{g}$ d'extrait) présentent des valeurs d'activités antioxydantes significativement supérieures $(\mathrm{P}<0,05)$ à celle de l'extrait éthanolique de $R$. officinalis $(2,54 \pm 0,06 \mu \mathrm{mol}$ $\mathrm{TE} / \mu \mathrm{g}$ d'extrait) Tableau 1 et la Figure 5.

La valeur de l'activité antioxydante du macéré aqueux de $U$. chamae $(3,04 \pm 0,20$ $\mu \mathrm{mol} \quad \mathrm{TE} / \mu \mathrm{g}$ d'extrait) est également supérieure à celle de l'extrait éthanolique de $R$. officinalis.

- Corrélation entre teneur en phénols totaux et activité antioxydante (ORAC)

Les résultats ont montré l'existence d'une corrélation linéaire $\left(R^{2}\right)$ significative $(P<0,05)$ entre la teneur des phénols totaux et activité antioxydante (ORAC) des extraits de $U$. chamae à raison de 93,73\% (Figure 6).

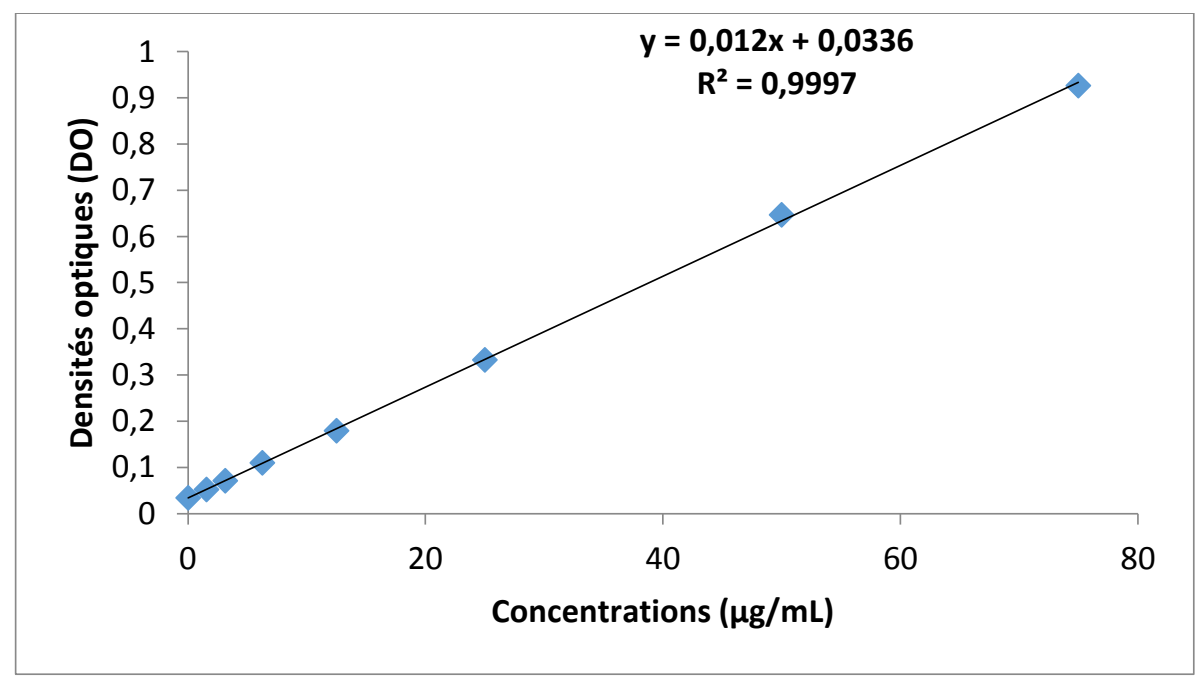

Figure 1 : Courbe d'étalonnage d'acide gallique. 


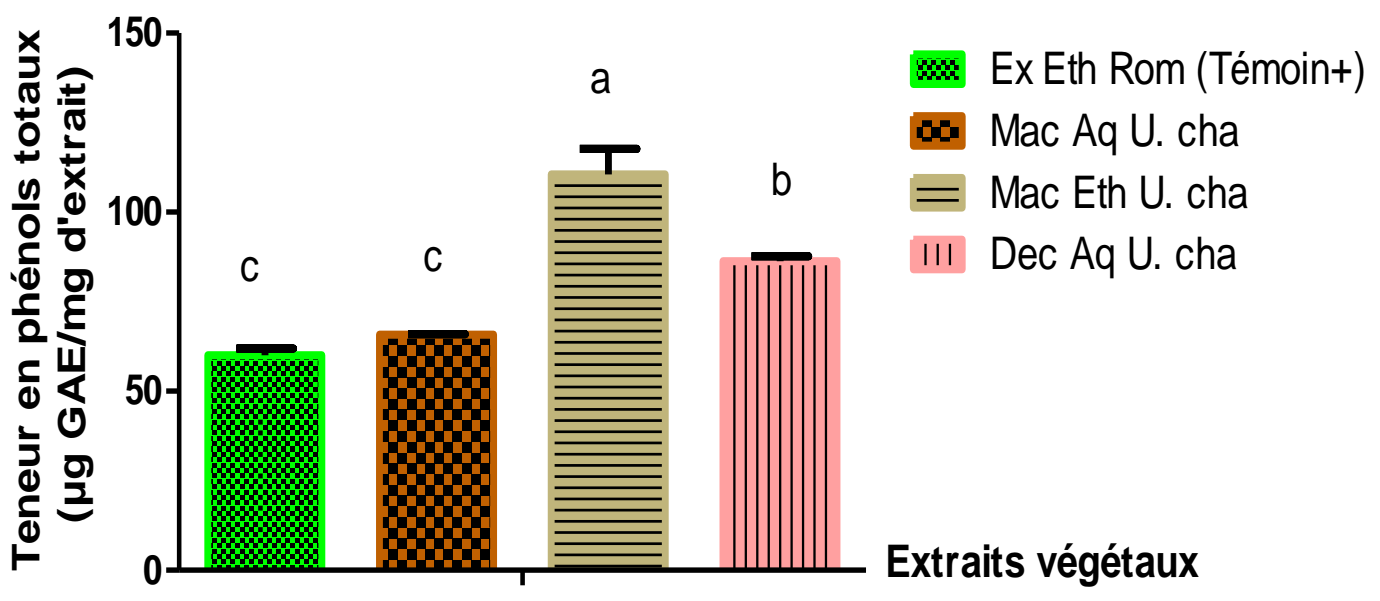

Figure 2: Teneur en phénols totaux des extraits de Uvaria chamae et de l'extrait éthanolique de Romarin officinalis. Les barres avec les lettres différentes indiquent des teneurs significativement différentes $(\mathrm{P}<0,05)$. Chaque valeur représente la moyenne \pm SEM de trois essais. Ex Eth Rom : Extrait éthanolique de Romarin officinalis ; Mac Aq U. cha : Macéré aqueux de Uvaria chamae; Mac Eth U. cha : Macéré éthanolique de Uvaria chamae; Dec Aq U. cha : Décocté aqueux de Uvaria chamae

Tableau 1 : Capacité anti-oxydante des extraits de $U$. chamae, de $R$. officinalis et de l'Acide chlorogénique.

\begin{tabular}{|c|c|c|c|c|c|}
\hline \multirow[t]{2}{*}{ Plantes } & \multirow[t]{2}{*}{ Extraits } & \multirow{2}{*}{$\begin{array}{c}\text { TPC } \\
\text { ( } \mu \text { g GAE/mg } \\
\text { d'extrait) }\end{array}$} & \multicolumn{2}{|c|}{ DPPH } & \multirow[t]{2}{*}{$\begin{array}{c}\text { ORAC } \\
(\mu \mathrm{mol} \text { TE } / \mu \mathrm{g} \text { d'extrait) }\end{array}$} \\
\hline & & & $\begin{array}{c}\text { IC50 } \\
(\mathrm{mg} / \mathrm{mL})\end{array}$ & $\begin{array}{c}\text { TE }(\mu \mathrm{mol} \text { TE/g } \\
\text { d'extrait) }\end{array}$ & \\
\hline \multirow{3}{*}{$\begin{array}{l}\text { Uvaria } \\
\text { chamae }\end{array}$} & Mac Aq & $65,80 \pm 0,13$ & $>1$ & $203,14 \pm 15,90$ & $3,04 \pm 0,20$ \\
\hline & Mac Eth & $110,51 \pm 7,18$ & $0,08 \pm 0,02$ & $371,26 \pm 18,42$ & $3,51 \pm 0,15$ \\
\hline & Dec Aq & $86,34 \pm 1,38$ & $>1$ & $224,39 \pm 18,39$ & $3,36 \pm 0,18$ \\
\hline Romarin & Extrait & & & & \\
\hline$(\mathrm{T}+)$ & Eth & $60,09 \pm 1,90$ & $0,32 \pm 0,02$ & $261,30 \pm 18,12$ & $2,54 \pm 0,06$ \\
\hline \multicolumn{2}{|c|}{ Acide chlo $(\mathrm{T}+)$} & nd & nd & $3236,57 \pm 305,97$ & $\begin{array}{l}4,07 \pm 0,37 \mu \text { mol } \\
\text { TE } / \mu \mathrm{mol} \text { d'acide chlo }\end{array}$ \\
\hline
\end{tabular}

Chaque valeur représente la moyenne \pm SEM de trois essais. Ex Eth Rom $(\mathbf{T}+)$ : Extrait éthanolique de Romarin officinalis (Témoin positif), Mac Aq : Macéré aqueux, Mac Eth : Macéré éthanolique, Dec Aq : Décocté aqueux, Acide chlo (T+) : Acide chlorogénique (Témoin positif), TPC : Teneur en Phénols totaux, ORAC : Oxygen Radical Antioxydant Capacity, DPPH : 2,2-diphényl-1-picrylhydrazyl, CI50 : Concentration inhibitrice, TE : équivalent Trolox, nd : non déterminé 


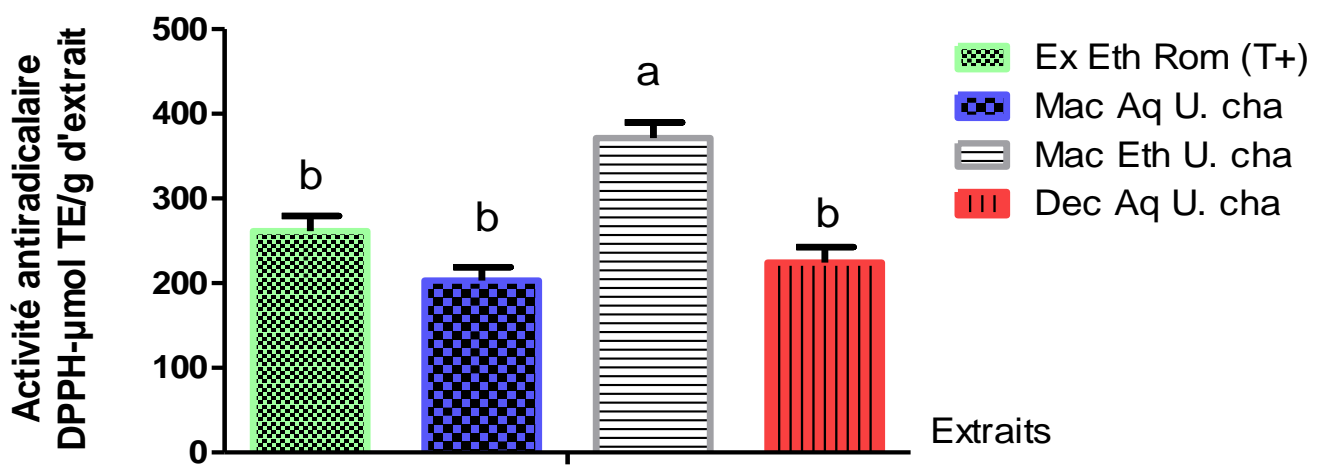

Figure 3: Activité antiradicalaire des extraits de $U$. chamae et de l'extrait éthanolique de $R$. officinalis. Les barres avec les lettres différentes indiquent des activités significativement différentes $(\mathrm{P}<0,05)$. Chaque valeur représente la moyenne \pm SEM de trois essais.

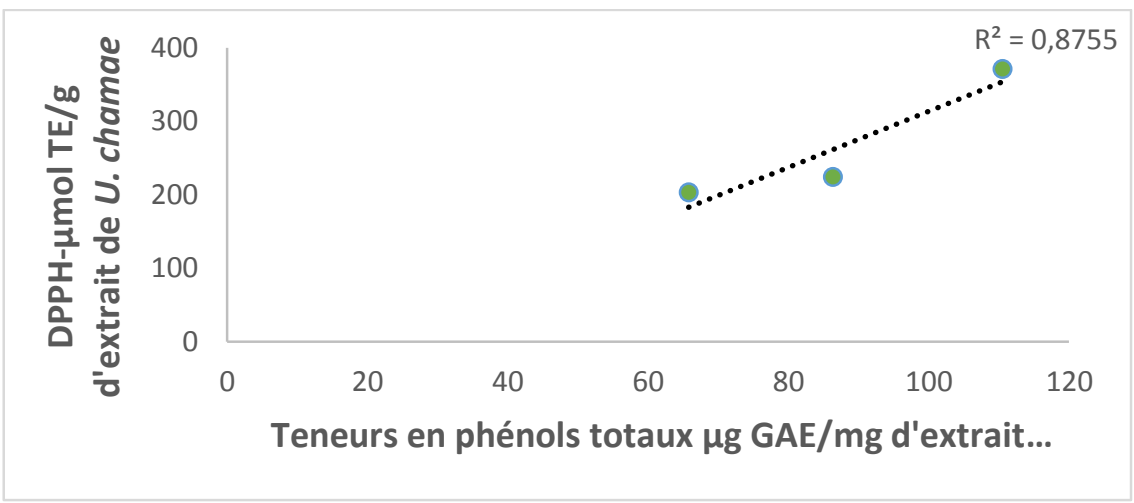

Figure 4 : Corrélation linéaire entre activité antiradicalaire (DPPH) et teneur en phénols totaux des extraits de $U$. chamae.

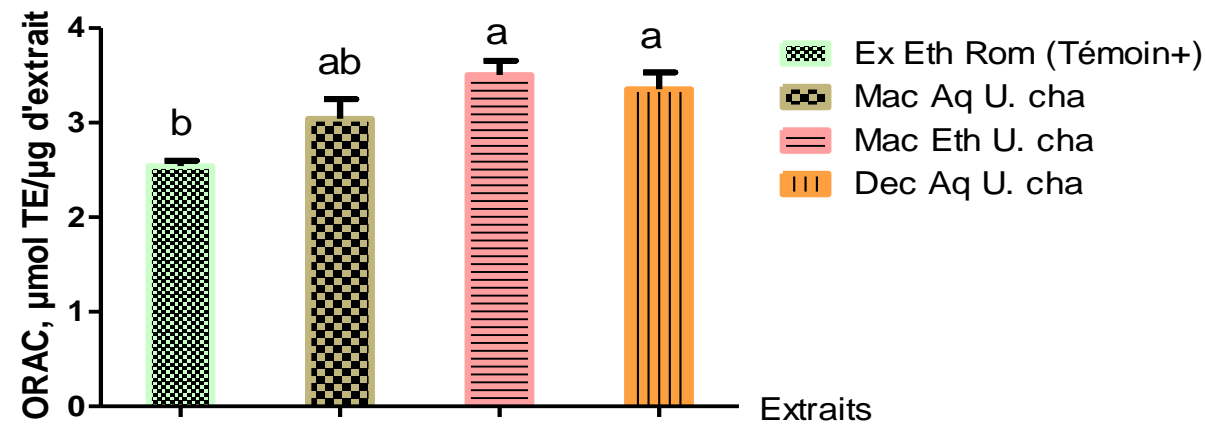

Figure 5 : Activité antioxydante des extraits de $U$. chamae et de l'extrait éthanolique de $R$. officinalis par le test ORAC. Les barres avec les lettres différentes indiquent des activités significativement différentes $(P<0,05)$. Chaque valeur représente la moyenne \pm SEM de trois essais. Ex Eth Rom : Extrait éthanolique de Romarin officinalis; Mac Aq U. cha : Macéré aqueux de Uvaria chamae; Mac Eth U. cha : Macéré éthanolique de Uvaria chamae ; Dec Aq U. cha : Décocté aqueux de Uvaria chamae. 


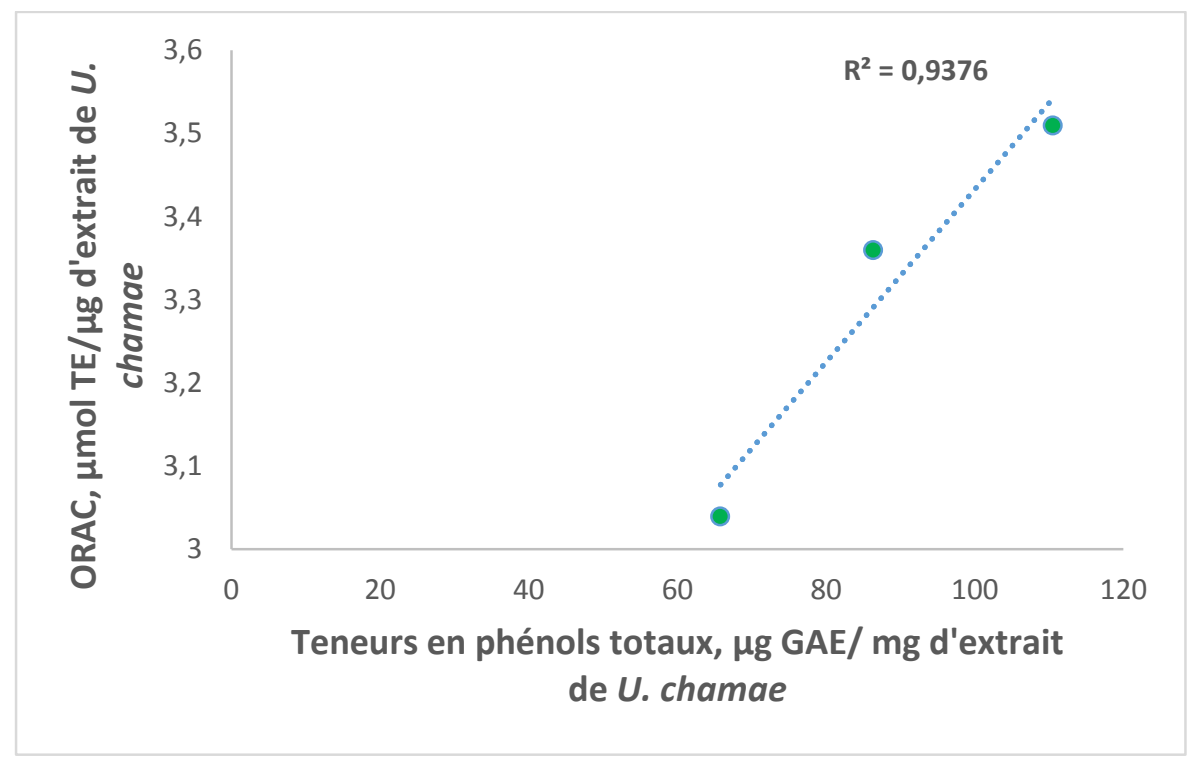

Figure 6 : Corrélation linéaire entre activité antioxydante (Test ORAC) et phénols totaux des extraits de $U$. chamae.

\section{DISCUSSION}

Le traitement de certaines pathologies dues au stress oxydatif est un enjeu majeur de santé publique qui nécessite l'utilisation de nouvelles substances antioxydantes naturelles ayant des effets bénéfiques. Parmi ces substances, l'extrait éthanolique de la plante de référence Rosmarinus officinalis (Romarin) considérée par l'Union Européenne comme un antioxydant alimentaire (E 392) (European Commission, 2010), a présenté une teneur en phénols totaux de l'ordre de $60,09 \pm 1,90 \mu \mathrm{g}$ GAE/mg d'extrait.

En outre, l'étude indique que $U$. chamae enregistre des teneurs élevées en phénols totaux $(110,51 \pm 7,18 \mu \mathrm{g} \mathrm{GAE} / \mathrm{mg}$ d'extrait) pour le macéré éthanolique suivi du décocté aqueux (86,34 $\pm 1,38 \mu \mathrm{g} \mathrm{GAE} / \mathrm{mg}$ d'extrait) et du macéré aqueux $(65,80 \pm 0,13 \mu \mathrm{g}$ GAE$/ \mathrm{mg}$ d'extrait).

Ces différences de teneurs chez ces espèces végétales pourraient être dues à la composition phénolique des extraits (Hayouni et al., 2007), aux solvants utilisés, à la méthode d'extraction ainsi qu'à la température d'extraction (Ayoola et al., 2006 ; Eva et al., 2016).

Nos résultats, en indiquant également que l'éthanol permet une meilleure extraction des polyphénols totaux, confirment ceux de Koné et al. (2015). Selon cet auteur, l'extrait éthanolique enregistre une teneur plus élevée $(21,14 \pm 0,51 \mathrm{mg} \mathrm{GAE} / \mathrm{g}$ d'extrait $)$ que l'extrait aqueux (8,92 $\pm 0,92 \mathrm{mg} \mathrm{GAE} / \mathrm{g}$ d'extrait).

D'autres études ont montré que les facteurs géographiques, climatiques et génétiques, mais aussi le degré de maturation de la plante, l'espèce, l'organe, les conditions de séchage et la durée de stockage ont une forte influence sur la teneur en polyphénols des espèces végétales (Alain et al., 2011; Merouane et al., 2014 ; El Hazzat et al., 2015).

Cependant, de faibles teneurs en phénols totaux des extraits de racines de $U$. chamae $\left(0,10 \pm 0,30 \mathrm{mg} 100 \mathrm{~g}^{-1}\right)$ sont observées par Okwu \& Iroabuchi (2009). En effet, la solubilité des polyphénols est liée aux caractéristiques du solvant utilisé, le rapport solvant / solide, leur degré de polymérisation ainsi que leur interaction avec d'autres constituants et la formation de complexes insolubles (Eva et al., 2016).

Les différents macérés et décoctés de ces plantes pourraient être de bon indicateurs d'activité antioxydante. En effet, la prévention des maladies par les polyphénols est principalement due à leurs pouvoirs antioxydants (Eva et al., 2016). Ces composés 
aident à rétablir le système immunitaire du corps en inhibant l'angiogenèse nécessaire à la croissance des tumeurs et agissent comme des agents anti-inflammatoires (Asensi et al., 2011; Tabrez et al., 2013). Ils possèdent également diverses activités biologiques telles que les activités anticoagulantes, antiallergiques, antiinflammatoires et vasodilatatrices (Gulcin et al., 2010). Ces effets thérapeutiques justifient la pertinence de l'utilisation traditionnelle de cette plante.

La méthode DPPH qui est basée sur une réaction de transfert d'électrons et la méthode de l'ORAC basée sur un transfert d'atomes d'hydrogènes qui complète la description antioxydante des différents macérés et décocté de cette plante étudiée (Morel et al., 2018). Il est donc conseillé d'utiliser plus d'une méthode pour mesurer la capacité antioxydante d'une substance afin de prendre en compte les différents mécanismes contribuant à l'action antioxydante. Les résultats des différentes méthodes sont dose-dépendantes. Comparé à l'antioxydant de référence (extrait éthanolique de $R$. officinalis) avec un CI50 de 0,32 $\pm 0,02$ $\mathrm{mg} / \mathrm{ml}$, le macéré éthanolique de $U$. chamae $(0,08 \pm 0,02 \mathrm{mg} / \mathrm{ml})$ est quatre fois plus actifs. Le macéré éthanolique de $U$. chamae exerce donc une activité antioxydante significative ( $\mathrm{P}$ $<0,05)$ vis-à-vis du radical DPPH.

En effet, la CI50 est inversement lié au pouvoir antioxydant d'un composé. Il exprime la quantité d'antioxydant requise pour réduire la concentration du radical libre de $50 \%$. Plus cette concentration (CI50) est faible, plus l'effet antioxydant est très élevé (Morel et al., 2018). Cette activité antiradicalaire significative de macéré éthanolique de $U$. chamae pourrait s'expliquer par sa richesse en phénols totaux. La CI50 de macéré éthanolique de $U$. chamae de notre étude est supérieure à celle de l'extrait de $U$. chamae $(4,02 \pm 0,50$ $\mu \mathrm{g} / \mathrm{ml}$ ) obtenue par Koné et al. (2015).

Par contre, le macéré aqueux de $U$. chamae ainsi que le décocté aqueux de $U$. chamae indiquent des activités antioxydantes moins significatives avec des valeurs de CI50 supérieures à $1 \quad \mathrm{mg} / \mathrm{ml}$. Ces activités antiradicalaires moins significatives $(\mathrm{P}>0,05)$ de ces extraits pourraient être liées par leurs teneurs moins importantes en phénols totaux qu'ils renferment. Aussi, ces différences d'activités antiradicalaires pourraient-elles s'expliquer par la distribution des composés bioactifs de ces extraits (Morel et al., 2018). Elles pourraient également être liées par la méthode d'extraction, le solvant utilisé et la structure chimique des composés bioactifs.

Par ailleurs, nos résultats montrent l'existence d'une bonne corrélation linéaire $\left(\mathrm{R}^{2}\right.$ $=0,8755)$ entre la teneur en phénols totaux et le pouvoir antiradicalaire des différents macérés et décocté des feuilles de $U$. chamae. Cette bonne corrélation linéaire suggère que pour cette plante, les composés phénoliques ayant des activités antithrombotiques, antiinflammatoires, hépatoprotectrices et anticancérigènes seraient responsables de l'activité antioxydante. Selon les travaux de N'guessan et al. (2007) et de Kagnou et al. (2020), il existe une corrélation linéaire entre la teneur en polyphénols totaux et l'activité antiradicalaire. Cela justifierait l'activité antiradicalaire significative de macéré éthanolique de $U$. chamae qui est riche en phénols totaux.

L'activité antioxydante de macéré aqueux, de macéré éthanolique et le décocté aqueux de $U$. chamae a également été évaluée par la méthode ORAC pour confirmation. Le macéré éthanolique de $U$. chamae $(3,51 \pm 0,15$ $\mu \mathrm{mol} \mathrm{TE} / \mu \mathrm{g}$ d'extrait) et le décocté aqueux de $U$. chamae $(3,36 \pm 0,18 \mu \mathrm{mol} \mathrm{TE} / \mu \mathrm{g}$ d'extrait $)$ présentent des valeurs d'activité antioxydante supérieures et significatives $(\mathrm{P}<0,05)$ à celle de l'extrait éthanolique de $R$. officinalis $(2,54 \pm$ $0,06 \mu \mathrm{mol} \mathrm{TE} / \mu \mathrm{g}$ d'extrait). Par contre, l'activité antioxydante du macéré aqueux de $U$. chamae $(3,04 \pm 0,20 \mu \mathrm{mol} \mathrm{TE} / \mu \mathrm{g}$ d'extrait), est légèrement supérieure à celle de l'extrait éthanolique de $R$. officinalis $(2,54 \pm 0,06 \mu \mathrm{mol}$ $\mathrm{TE} / \mu \mathrm{g}$ d'extrait) mais ne diffèrent pas significativement.

Ces différences de potentiel antioxydant à piéger les radicaux libres pourraient être dues à l'espèce végétale et à leur richesse en divers composés bioactifs. L'existence d'une corrélation linéaire $\left(\mathrm{R}^{2}\right)$ significative entre la teneur en phénols totaux et activité antioxydante (ORAC) des différents extraits de 
U. chamae à raison de $93,73 \%$, suggère que pour cette plante, les composés phénoliques seraient responsables de l'activité antioxydante observée.

La richesse en phénols totaux de nos extraits serait donc à la base de leur activité antioxydante. Des résultats similaires ont été rapportés par N'Guessan et al. (2007) et Morel et al. (2018). Selon ces auteurs, le pouvoir antioxydant d'un extrait de plante est lié à sa teneur en composés phénoliques. Ces activités antioxydantes pourraient également s'expliquer par la présence de groupement hydroxyle dans les composés phénoliques pouvant piéger les radicaux libres (Gulcin et al., 2010 ; Corrêa et al., 2018 ; Morel et al., 2018). Toutefois, une meilleure synergie et la diversité des composés des extraits pourraient aussi contribuer à cette bonne activité antioxydante (Eva et al., 2016).

\section{Conclusion}

Le dosage des phénols montre que les différents macérés et décocté des feuilles de $U$. chamae sont riches en phénols totaux. Les méthodes de DPPH et d'ORAC ont permis de mettre en évidence l'activité antioxydante des différents macérés et décocté de $U$. chamae. Les résultats montrent l'existence d'une bonne corrélation linéaire entre la teneur en phénols totaux et le pouvoir antioxydant des différents macérés et décocté de cette plante. Cette plante, $U$. chamae, de la pharmacopée ivoirienne, pourrait donc être considérée comme une source d'antioxydant naturel à des fins médicinales.

\section{CONFLIT D'INTERETS}

Les auteurs déclarent ne pas avoir de conflit d'intérêts.

\section{CONTRIBUTIONS DES AUTEURS}

$\mathrm{BB}$ a été l'expérimentateur principal. $\mathrm{CKDB}$ et $\mathrm{AO}$ ont activement participé à la rédaction du manuscrit. DND et RKK ont participé aussi à l'expérimentation. KO a supervisé les travaux et a participé à la rédaction du manuscrit.

\section{REMERCIEMENTS}

Les auteurs tiennent à remercier le Laboratoire de Pharmacologie et de Physiopathologie Expérimentale de la Faculté de Pharmacie de l'Université de Montpellier (France) pour son assistance technique et ses conseils.

\section{REFERENCES}

Adomou AC, Yedomonhan H, Djossa B, Legba SI, Oumorou M, Akoegninou A. 2012. Etude Ethnobotanique des plantes médicinales vendues dans le marché d'Abomey-Calavi au Bénin. International Journal of Biological and Chemical Sciences, 6(2): 745-772. DOI: https://doi.org/10.4314/ijbcs.v6i2.18

Alain HO, N'Guessan CEDD, Janat AM-B, Yves-Alain B. 2011. Teneurs en composés phénoliques de 10 plantes médicinales employées dans la tradithérapie de l'hypertension artérielle, une pathologie émergente en Côte d'Ivoire. Revue de génie industriel, 6 : 5561.

Asensi M, Ortega A, Mena S, Feddi F, Estrela JM. 2011. Natural polyphenols in cancer therapy. Critical Reviews in Clinical Laboratory Sciences, 48(5-6): 197-216. DOI: $10.3109 / 10408363.2011 .631268$

Ayoola GA, Sofidiya T, Odukoya O, Coker HAB. 2006. Phytochemical screening and free radical scavenging activity of some Nigerian medicinal plants. Journal of Pharmaceutical Sciences and Pharmacy Practice, 8: 133-136.

Bamba B, Golly KJ, Ouattara A, Kone M, Doukourou DN, Benie CKD, Kamou R, Ouattara K. 2019. Anti-inflammatory activity of the aqueous macerate of leaves of Uvaria chamae (P. Beauv) (Annonaceae) on acute edema of rat paw induced by carrageenan. International Journal of Pharmacognosy and Phytochemical Research, 11(2): 44-48. Doi: 10.25258/phyto.11.2.2

Corrêa WR, Serain AF, Aranha Netto L, Marinko JVN, Arena AC, Figueiredo de Santana Aquino D, Kuraoka-Oliveira AM, Junior AJ, Bernal LPT, Kassuya 
CAL, Salvador MJ. 2018. Antiinflammatory and antioxidant properties of the extract, tiliroside, and patuletin 3O- $\beta$-D-Glucopyranoside from Pfaffia townsendii (Amaranthaceae). Evidencebased Complementary and Alternative Medicine, 2018: 6057579. DOI: $10.1155 / 2018 / 6057579$

Davies MJ. 2004. Reactive species formed on proteins exposed to singlet oxygen. Photochemical and Photobiological Sciences, $\quad 3(1): \quad 17-25$. DOI: $10.1039 / \mathrm{b} 307576 \mathrm{c}$

El Hazzat N, Iraqi R, Bouseta A. 2015. Identification par GC-MS et GC-FID-O des composés volatils des olives vertes de la variété «Picholine marocaine »: Effet de l'origine géographique. International Journal of Biological and Chemical Sciences, 9(4): 2219-2233. DOI: $10.4314 / \mathrm{ijbcs} . \mathrm{v} 9 \mathrm{i} 4.40$

Etukudo I. 2003. Ethnobotany, conventional and traditional uses of plants, Verdict Press: Uyo, Nigeria, 191p. DOI: 10.12691/ajfst-5-5-3

European Commission. 2010. Commission Directive 2010/67/EU of 20 October 2010 amending Directive 2008/84/EC laying down specific purity criteria on food additives other than colours and sweeteners. Official Journal of the European Union. L 277: 17-19. https://eur-

lex.europa.eu/LexUriServ/LexUriServ.d o?uri=OJ:L:2010:277:0017:0026:EN:PD $\mathrm{F}$

Eva BM, Maša KH, Mojca Š, Željko K, Urban B. 2016. Polyphenols: Extraction methods, Antioxidative action, Bioavailability and Anticarcinogenic effects. Molecules, 21: 901. DOI: 10.3390/molecules21070901

Falleh H, Ksouri R, Chaieb K, KarrayBouraoui N, Trabelsi N, Boulaaba M, Abdelly C. 2008. Phenolic composition of Cynara cardunculus L. organs, and their biological activities. Comptes Rendus Biologies, 331: 372-379. DOI: 10.1016/j.crvi.2008.02.008
Favier A. 2003. Le stress oxydant. Intérêt conceptuel et expérimental dans la compréhension des mécanismes des maladies et potentiel thérapeutique. L'actualité Chimique, 269(270) : 108115.

https://www.lactualitechimique.org/Lestress-oxydant-interet-conceptuel-etexperimental-dans-la-comprehensiondes-mecanismes-des

Gulcin I, Huyut Z, Elmastas M, Aboul-Enein HY. 2010. Radical scavenging and antioxidant activity of tannic acid. Arabian Journal of Chemistry, 3: 43-53. https://doi.org/10.1016/j.arabjc.2009.12. 008

Hayouni E, Abedrabba M, Bouix M, Hamdi M. 2007. The effects of solvent and extraction method on the phenolic contents and biological activities in vitro of Tunisian Quecus coccifera L. and Juniperus phoenicea L. fruit extracts. Food Chemistry, 105(3): 1126-1134. DOI: 10.1016/j.foodchem.2007.02.010

Kagnou H, Sinalou O, Tchani GW, Sanvee S, Agbodan KA, Toundou O, Kpegba K. 2020. Etude phytochimique et activité antioxidante comparatives des trois variétés de Catharanthus roseus (L) G. Don. International Journal of Biological and Chemical Sciences, 14(6): 23522361.

DOI: https://dx.doi.org/10.4314/ijbcs.v14i6.33

Kone M, Ouattara K, Gnahoue G, Ouattara A, Coulibaly A. 2013. Study Ethnopharmacological and Phytochemical Screening of Some Plants Involved in the Treatment of Abdominal Infections in The Department of Kouto (COTE D'IVOIRE). Scholars Journal of Applied Medical Sciences, 1(2):56-61. http://saspublisher.com/wpcontent/uploads/2013/04/SJAMS125661.pdf

Koné M, Toure A, Ouattara K, Coulibaly A. 2015. Phytochemical Composition, Antioxydant and Antibacterial Activities of Root of Uvaria chamae P. Beauv. (Annonacea) Used in Treatment of Dysentery in North of Côte d'Ivoire. 
International Journal of Pharmacognosy and Phytochemical Research, 7(6): 10471053.

https://www.researchgate.net/publication /290450088_Phytochemical_compositio n_antioxidant_and_antibacterial_activiti es_of_root_of_Uvaria_chamae_p_Beauv _Annonaceae_used_in_treatment_of_dys entery_in_north_of_Cote_d'ivoire

Maiese K, Chong ZZ. 2007. Mechanistic insights into diabetes mellitus and oxidative stress. Current Medicinal Chemistry, 14: 1729-1738. DOI: $10.2174 / 092986707781058968$

Melo A, Monteiro L, Lima RM, Oliveira DM, Cerqueira MD, EL-Bacha RS. 2011. Oxidative stress in neurodegenerative diseases: mechanisms and therapeutic perspectives. Oxidative Medicine of Cellular and Longevity : $14 \mathrm{p}$. DOI: $10.1155 / 2011 / 467180$

Merouane A, Noui A, Medjahed H, Nedjari BAK, Saadi A. 2014. Activité antioxydante des composés phénoliques d'huile d'olive extraite par méthode traditionnelle. International Journal of Biological and Chemistry Sciences, 8(4): 1865-1870. DOI: 10.4314/ijbcs.v8i4.45

Morel S, Arnould S, Vitou M, Boudard F, Guzman C, Poucheret P, Fons F, Rapior S. 2018. Antiproliferative and antioxidant activities of wild Boletales mushrooms from France. International Journal of Medicinal Mushrooms, 20(1): 13-29. DOI: 10.1615/IntJMedMushrooms.2018 025329

Moses SO, Olowu R, Adewale NSD, William NS. 2013. 1-Nitro- 2-phenylethane dominates the chemical composition of the leaf essential oil of Uvaria chamae from Badagry, Nigeria. American Journal of Essential Oils and Natural Products, 1(1): $\quad 48-50 . \quad$ DOI: https://www.essencejournal.com/pdf/201 3/vollissue1/PartA/15-352.pdf

N'guessan JD, Zirihi GN, Kra AKM, Kouakou K, Djaman AJ, Guede-guina F. 2007. Free radical scavenging activity, flavonoid and phenolic contents of selected Ivoirian plants. International
Journal of Natural and Applied Sciences, 4: 425-429. DOI: 10.4314/ijonas.v3i4.36155

Niki E. 2009. Lipid peroxidation: physiological levels and dual biological effects. Free Radical Biology \& Medicine, 47(5): 469-484. DOI: 10.1016/j.freeradbiomed.2009.05.032

Nwosu M. 2000. Plant resources used by women as herbal medicines and cosmetics in Southeastern Nigeria. Arztezeitschrift for Naturopathy, 41(11): 760-767.

https://www.researchgate.net/publication /289349305_Plant_resources_used_by_tr aditional_women_as_herbal_medicines_ and_cosmetics_in_Southeast-Nigeria

Okwu DE, Iroabuchi F. 2009. Phytochemical Composition and Biological Activities of Uvaria chamae and Clerodendoron splendens. E-Journal of Chemistry, 6(2): 553-560.

DOI: https://doi.org/10.1155/2009/190346

Olakunle O, Kassim ML, Biaffra E, Andrew G, Henrietta A, Victor RG. 2005. Effects of Root Extracts of Fagara zanthoxyloides on the In Vitro Growth and Stage Distribution of Plasmodium falciparum. American Society for Microbiology, 49: 264-268. DOI: $10.1128 /$ aac.49.1.264268.2005

Omale J, Ebiloma UG, Idoko GO. 2013. Uvaria chamae (Annonaceae) plant extract neutralizes some biological effects of Naja nigricollis Snake venom in rats. Bristish Journal of Pharmacology and Toxicology $\quad 4(2): \quad 41-50$. https://www.researchgate.net/publication /310400000_Omale_J_Ebiloma_UG_Ido ko_GO_2013_Uvaria_chamae_Annonac eae_Plant_Extract_Neutralizes_Some_Bi ological_Effects_of_Naja_nigricollis_Sn ake_Venom_in_Rats_British_Journal_of _Pharmacology_and_Toxicology_42_41

Panicker VP, George S, Dhanush KB. 2014. Toxicity study of butylated hydroxyl toluene (BHT) in rats. World Journal of Pharmacy and Pharmaceutical Sciences, 3(8): 758-763. https://www.researchgate.net/publication 
1304675202_Toxicity_study_of_butylate d_hydroxyl_toluene_BHT_in_rats

Popoola TD, Awodele O, Omisanya A, Obi N, Umezinwa C, Fatokun AA. 2016. Three indigenous plants used in anti-cancer remedies, Garcinia kola Heckel (stem bark), Uvaria chamae P. Beauv. (root) and Olax subscorpioidea Oliv. (root) show analgesic and anti-inflammatory activities in animal models. Journal of Ethnopharmacology, 194: 440-449. DOI: $10.1016 /$ j.jep.2016.09.046

Sanchez C. 2017. Reactive oxygen species and antioxidant properties from mushrooms. Synthetic and Systems Biotechnology, 2: 13-22.

DOI: https://doi.org/10.1016/j.synbio.2016.12. 001

Tabrez S, Priyadarshini M, Urooj M, Shakil S, Ashraf GM, Khan MS, Kamal MA, Alam Q, Jabir NR, Abuzenadah AM,
Chaudhary AGA, Damanhouri GA. 2013. Cancer Chemoprevention by Polyphenols and Their Potential Application as Nanomedicine. Journal of Environmental Science and Health, 31: 67-98. DOI: 10.1080/10590501.2013.763577

Vincent HK, Innes KE, Vincent KR. 2007. Oxidative stress and potential interventions toreduce oxidative stress in overweight and obesity. Diabetes, Obesity and Metabolism, 9: 813-839. DOI: 10.1111/j.1463-1326.2007.00692.x Zirihi GN, Kra AKM, Bahi C, Guédé-Guina F. 2003. Evaluation de l'activité antifongique de Microglossa pirifolia (lamarck) o. kuntze (Asteraceae) «PYMI » sur la croissance in vitro de Candida albicans. Revue de Médecine et Pharmacopée Africaine, 17 : 11-18. DOI: 10.19044/esj.2016.v12n3p171. 